\title{
BMJ Open Prevalence and risk factors of metabolic- associated fatty liver disease during 2014-2018 from three cities of Liaoning Province: an epidemiological survey
}

\author{
Lin Guan, ${ }^{1}$ Xinhe Zhang, ${ }^{1}$ Haoyu Tian, ${ }^{2}$ Xing Jin, ${ }^{1}$ Hang Fan, ${ }^{3}$ Ningning Wang, ${ }^{1}$ \\ Jing Sun, ${ }^{1}$ Dan Li, ${ }^{1}$ Jia Li, ${ }^{3}$ Xue Wang, ${ }^{1}$ Zilu Zeng, ${ }^{1}$ Yiling Li (D) ${ }^{1}$
}

To cite: Guan L, Zhang $X$, Tian $\mathrm{H}$, et al. Prevalence and risk factors of metabolicassociated fatty liver disease during 2014-2018 from three cities of Liaoning Province: an epidemiological survey. BMJ Open 2022;12:e047588. doi:10.1136/ bmjopen-2020-047588

- Prepublication history for this paper is available online. To view these files, please visit the journal online (http://dx.doi org/10.1136/bmjopen-2020047588).

$L G$ and $X Z$ contributed equally.

$L G$ and $X Z$ are joint first authors.

Received 02 December 2020 Accepted 02 February 2022

Check for updates

(C) Author(s) (or their employer(s)) 2022. Re-use permitted under CC BY-NC. No commercial re-use. See rights and permissions. Published by BMJ.

${ }^{1}$ Gastroenterology Department, The First Hospital of China

Medical University, Shenyang, Liaoning, China

${ }^{2}$ The 3rd Clinical Department, China Medical University, Shenyang, Liaoning, China ${ }^{3}$ Data Operation and Management Department, Neusoft Corporation, Shenyang, China

Correspondence to Dr Yiling Li; lyl-72@163.com

\section{ABSTRACT}

Objective To investigate the incidence and characteristics of metabolic-associated fatty liver disease (MAFLD) in individuals undergoing physical examination in Liaoning Province (China).

Design Retrospective study.

Setting Single centre.

Participants Adults who underwent routine health examination at Xikang Medical Center in Liaoning Province (Shenyang, Dandong and Dalian cities) between January 2014 and December 2018.

Results Among the 204394 included subjects, 71756 were diagnosed with MAFLD, accounting for $35.28 \%$. The total prevalence of MAFLD in Shenyang, Dandong and Dalian cities over the past 5 years was $35.8 \%$, $40.41 \%$ and $31.7 \%$, respectively. Men had a prevalence of $46.12 \%$, which was higher than in women $(21.80 \%)$. The percentage of MAFLD in body mass index $(\mathrm{BMI})<23 \mathrm{~kg} /$ $\mathrm{m}^{2}$ and $\geq 23 \mathrm{~kg} / \mathrm{m}^{2}$ was $6.49 \%$ and $53.23 \%$, respectively. In all subjects, BMI, systolic blood pressure, diastolic blood pressure (DBP), fasting blood glucose (FBG), triglyceride (TG), total cholesterol (TC), low-density lipoprotein cholesterol (LDL-C), high-density lipoprotein cholesterol (HDL-C), alanine transaminase, aspartate transaminase (AST), alkaline phosphatase (ALP), $\gamma$-glutamyl transferase (GGT), blood urea nitrogen, serum creatinine (SCr), serum uric acid (SUA), haematocrit (HCT), mean corpuscular volume (MCV) and urine protein were independently associated with MAFLD (all $p<0.001$ ). In lean subjects, DBP, FBG, TG, TC, LDL-C, HDL-C, AST, ALP, GGT, SCr, SUA, $\mathrm{HCT}$ and MCV were independently associated with MAFLD (all $p<0.001$ ).

Conclusion The prevalence of MAFLD in Liaoning Province was found to be associated with sex, cities with different economic statuses, BMl and multiple metabolic indicators.

\section{INTRODUCTION}

Non-alcoholic fatty liver disease (NAFLD) is one of the most common causes of chronic liver disease and is defined as the presence of $\geq 5 \%$ hepatic steatosis (HS). ${ }^{12}$ Recently, it was recommended to rename NAFLD to metabolic-associated fatty liver disease (MAFLD), which might increase awareness
Strengths and limitations of this study

- The study is a large-scale epidemiological survey.

- This is one of the first epidemiological studies after non-alcoholic fatty liver disease was renamed to metabolic-associated fatty liver disease (MAFLD).

- The study provides time trends from three cities in China with varying economic development.

- There is lack of data on MAFLD patients with type 2 diabetes mellitus in the study.

- Although majority of the metabolic risk factors have been discussed in the study, further comprehensive assessment is required.

of the disease and reduce stigma. ${ }^{34}$ NAFLD is associated with chronic diseases, such as insulin resistance and/or type 2 diabetes mellitus (T2DM), dyslipidaemia, hypertriglyceridaemia and hypertension. ${ }^{5-8}$ The prevalence of NAFLD was reported to be $24 \%-45 \%$, with an estimated prevalence of $76 \%$ in patients with T2DM. $^{9}$ In non-obese patients, NAFLD is associated with elevated triglyceride (TG) level, enlarged waist circumference and insulin resistance. ${ }^{10}$

Chinese individuals are at substantially higher risk of NAFLD, even those with noticeably lower body mass index (BMI, $\mathrm{kg} / \mathrm{m}^{2}$ ) values, compared with the US population. ${ }^{11}$ Factors such as waist circumference, T2DM, increased TG level, low high-density lipoprotein cholesterol (HDL-C) level and metabolic syndrome are known as predictive factors for NAFLD in adults, of which metabolic syndrome is considered a strong predictive factor. ${ }^{12} 13$ NAFLD is also associated with dyslipidaemia characterised by high TG, high low-density lipoprotein cholesterol (LDL-C) and low HDL-C levels. ${ }^{14}$ Determining the risk factors associated with a worse prognosis is essential to develop further effective therapeutic strategies. 
Since the 21st century, the prevalence of NAFLD in China has significantly increased to reach about one in three mainland Chinese residents. ${ }^{12} \mathrm{~A}$ recent metaanalysis showed that the incidence of NAFLD is higher in northern China (35.78\%) and lower in northwestern China (21.52\%). ${ }^{15}$ Among provinces in northern China, Heilongjiang has the highest incidence, with up to $50.48 \% .{ }^{15}$ Nevertheless, the results might be biased due to the small number of studies, and the incidence of NAFLD in northern China remains significantly higher than in the southern provinces. In addition, the risk of NAFLDrelated mortality has also increased significantly, mainly due to liver fibrosis-associated diseases. ${ }^{16}$ NAFLD has become an important public health concern, negatively influencing the Chinese population as well as increasing the socioeconomic burden. ${ }^{11} 1215$ Therefore, Chinese medical professionals and stakeholders urgently need to develop further accurate early diagnostic methods for NAFLD. Multiple studies have reported that NAFLD is a heterogeneous entity, and its development is related to sex, age, race, mild to moderate alcohol consumption, dietary intake, lifestyle, obesity, metabolism, genetic variations and educational level. ${ }^{11} 121516$ With uneven economic development and diverse lifestyles among the different provinces in China, the epidemiology of NAFLD has shown remarkable regional differences. With understanding the epidemiology of NAFLD in Liaoning Province (China), we can conduct targeted education and clinical research for precise prevention and control of NAFLD. Moreover, after NAFLD was renamed to MAFLD, few studies have investigated the prevalence of MAFLD.

There have been no data from large-scale epidemiological investigations of MAFLD in Liaoning Province over the past 10 years. Therefore, the present retrospective study aimed to investigate the incidence and disease characteristics of MAFLD in Liaoning Province. Physical examination data of residents of three cities (Shenyang, Dandong and Dalian) in Liaoning Province with different economic levels were collected from 2014 to 2018.

\section{METHODS}

\section{Study subjects}

This is a retrospective study of adults who underwent routine health examination at Xikang Medical Center in Liaoning Province (including clinics in Shenyang, Dandong and Dalian) between January 2014 and December 2018.

The three cities are from different parts of Liaoning Province (north, south and east; figure 1) and have different economic levels. Shenyang, located in the north of Liaoning Province, is a highly developed inland city, while Dalian in the south of Liaoning Province is a developed coastal city. Dandong, in the east of Liaoning Province, is a poorly developed city bordering North Korea.

The inclusion criteria were as follows: (1) patients aged $>18$ years old; (2) patients who have lived in Shenyang, Dalian or Dandong for at least 5 years; (3) participation

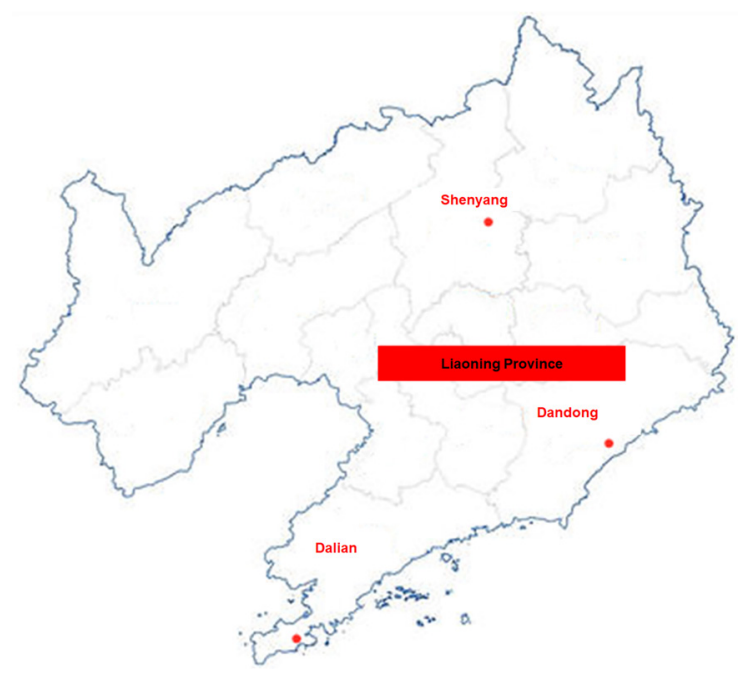

Figure 1 Location of the three study cities in Liaoning Province.

in annual physical examination; and (4) no missing data (as listed in table 1). The exclusion criteria were as follows: (1) liver cirrhosis; (2) liver cancer; (3) any liver lesions; or (4) no ultrasound examination. For participants undergoing more than one examination during the study period, only the first examination was included in this study. According to the guidelines, the diagnosis of MAFLD is no longer an exclusive diagnosis. Therefore, in diagnosed patients, conditions such as alcohol consumption and other liver diseases were not ruled out

The selected patients were divided into the MAFLD group and the non-MAFLD group based on ultrasound evidence of HS, in addition to one of the following two criteria, namely overweight/obesity (BMI $\geq 23 \mathrm{~kg}$ / $\mathrm{m}^{2}$, according to the standards recommended by the WHO for Asians, ${ }^{17}$ or BMI $<23 \mathrm{~kg} / \mathrm{m}^{2}$, with at least two evidence of metabolic dysregulation, such as blood pressure $\geq 130 / 85 \mathrm{~mm} \mathrm{Hg}, \quad \mathrm{TG} \geq 1.70 \mathrm{mmol} / \mathrm{L}$, and HDL-C $<1.0 \mathrm{mmol} / \mathrm{L}$ for men and $<1.3 \mathrm{mmol} / \mathrm{L}$ for women).$^{18}$ Regrettably, 3156 patients with T2DM who received medical intervention were not included in this study due to serious incomplete medical records.

\section{Patient and public involvement}

No patients were involved.

\section{Physical examination}

All physical examinations in this study are part of routine examination. Blood pressure measurements, including systolic blood pressure (SBP) and diastolic blood pressure (DBP), were performed twice after participants have seated, in a calm state for at least 5 min, using an electronic sphygmomanometer (HEM-7200; Omron Healthcare, Kyoto, Japan). Height and weight were measured in the morning on an empty stomach.

\section{Laboratory examination}

All physical blood tests in this study are part of routine examination. Anterior cubital vein blood was drawn in 
Table 1 Characteristics of patients with and without MAFLD

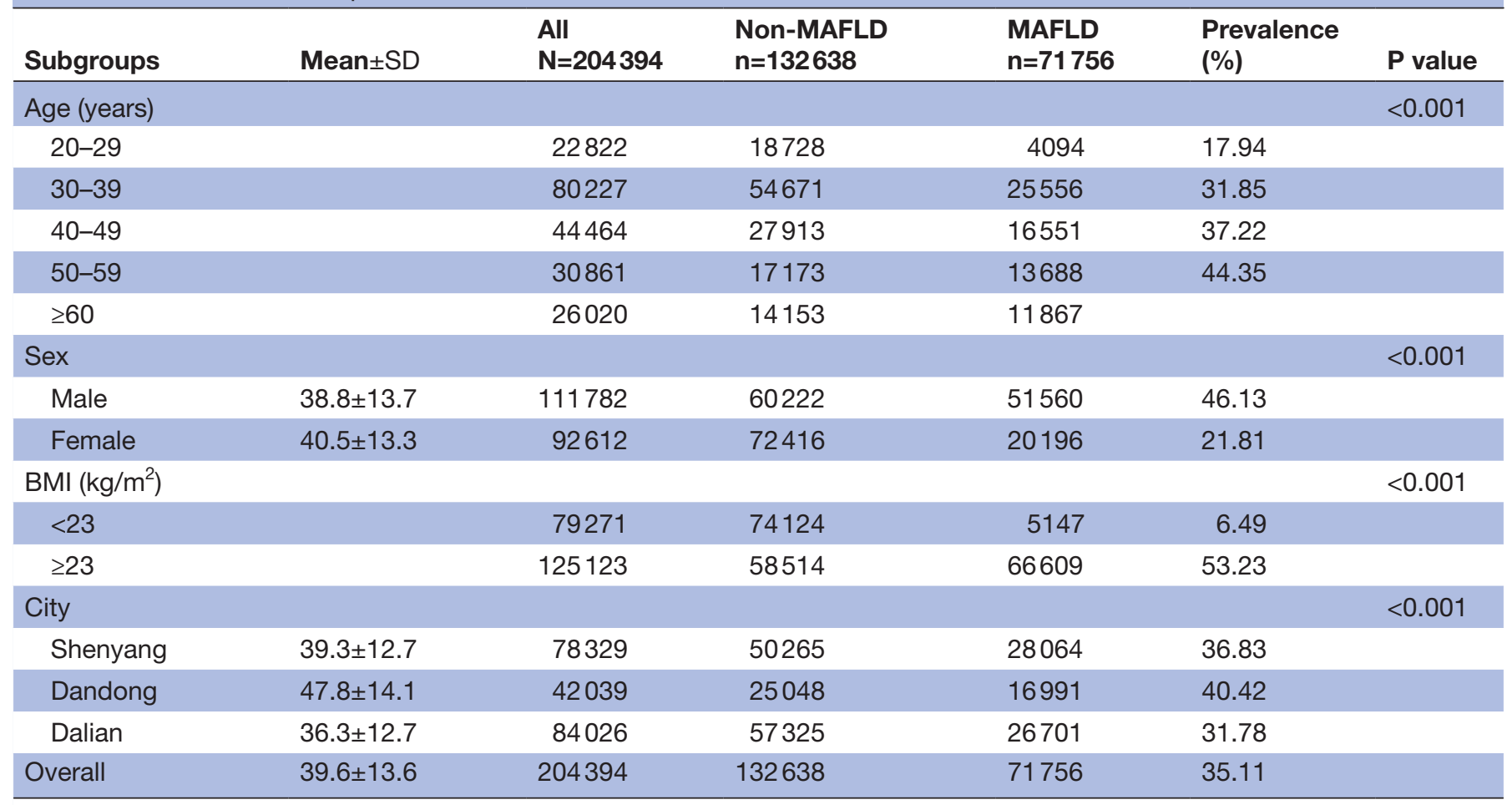

BMI, body mass index; MAFLD, metabolic-associated fatty liver disease.

fasting state (at least 8 hours). A mid-course morning urine specimen was taken as well. Routine blood panel, liver function, kidney function, serum uric acid (SUA) level, fasting blood glucose (FBG) level, blood lipids and routine urine analysis were assessed using a 7600 Autoanalyzer (Hitachi, Tokyo, Japan).

\section{Colour Doppler ultrasound of the liver and gall bladder}

A liver ultrasound is part of routine examination and was performed by two experienced ultrasound radiologists with at least 5 years of experience using an IU22 system (Philips Healthcare, Best, The Netherlands). A participant was diagnosed with HS when the ultrasound examination showed that the liver had fatty liver changes (hyperechogenicity due to increased acoustic interface caused by the intracellular accumulation of lipid vesicles, blurring of vascular margins, enlarged liver size and increased acoustic attenuation ${ }^{10}$ ).

\section{Statistical analysis}

R V.3.5.3 and R Commander V.2.5-3 were used for statistical analysis. Categorical data were expressed as n (\%) and were analysed using $\chi^{2}$ test. Continuous variables conforming to normal distribution (according to the Kolmogorov-Smirnov test) were expressed as mean \pm SD and were analysed using Student's t-test. Abnormally distributed continuous variables were presented as median (IQR) and were analysed using Mann-Whitney $U$ test. Factors associated with MAFLD were identified using univariate analysis. Variables with $\mathrm{p}<0.05$ were included in a multivariate logistic regression model. $\mathrm{P}<0.05$ was considered statistically significant.

\section{RESULTS}

Characteristics of the subjects

A total of 284129 subjects were examined during the study period and 204394 met the eligibility criteria. Table 1 presents the characteristics of the subjects. The subjects' mean age was $39.6 \pm 13.6$ years old. The number of male and female subjects was 111782 and 92 612, respectively. The male subjects' mean age was $38.8 \pm 13.7$ years old and that of female subjects was $40.5 \pm 13.3$ years old. Shenyang included 78329 subjects, aged 39.3 \pm 12.7 years old. No routine health examination was performed in Dandong in 2014. Dandong included 42039 subjects, aged $47.8 \pm 14.1$ years old. Finally, 84026 subjects were from Dalian, aged $36.3 \pm 12.7$ years old.

\section{Prevalence of MAFLD among the healthy population}

Among the 204394 included subjects, 71756 were diagnosed with MAFLD, accounting for $35.28 \%$ (table 1). The prevalence of MAFLD increased with age $(p<0.001)$, which was higher in men than in women $(p<0.001)$, higher in overweight/obese subjects than in lean ones $(\mathrm{p}<0.001)$, and higher in Dandong, followed by Shenyang and Dalian $(\mathrm{p}<0.001)$ (table 1$)$. The prevalence of MAFLD in Shenyang, Dandong and Dalian over the past 5 years was $36.83 \%, 40.42 \%$ and $31.78 \%$, respectively. 
Table 2 Changes in the prevalence of MAFLD over time (2014-2018)

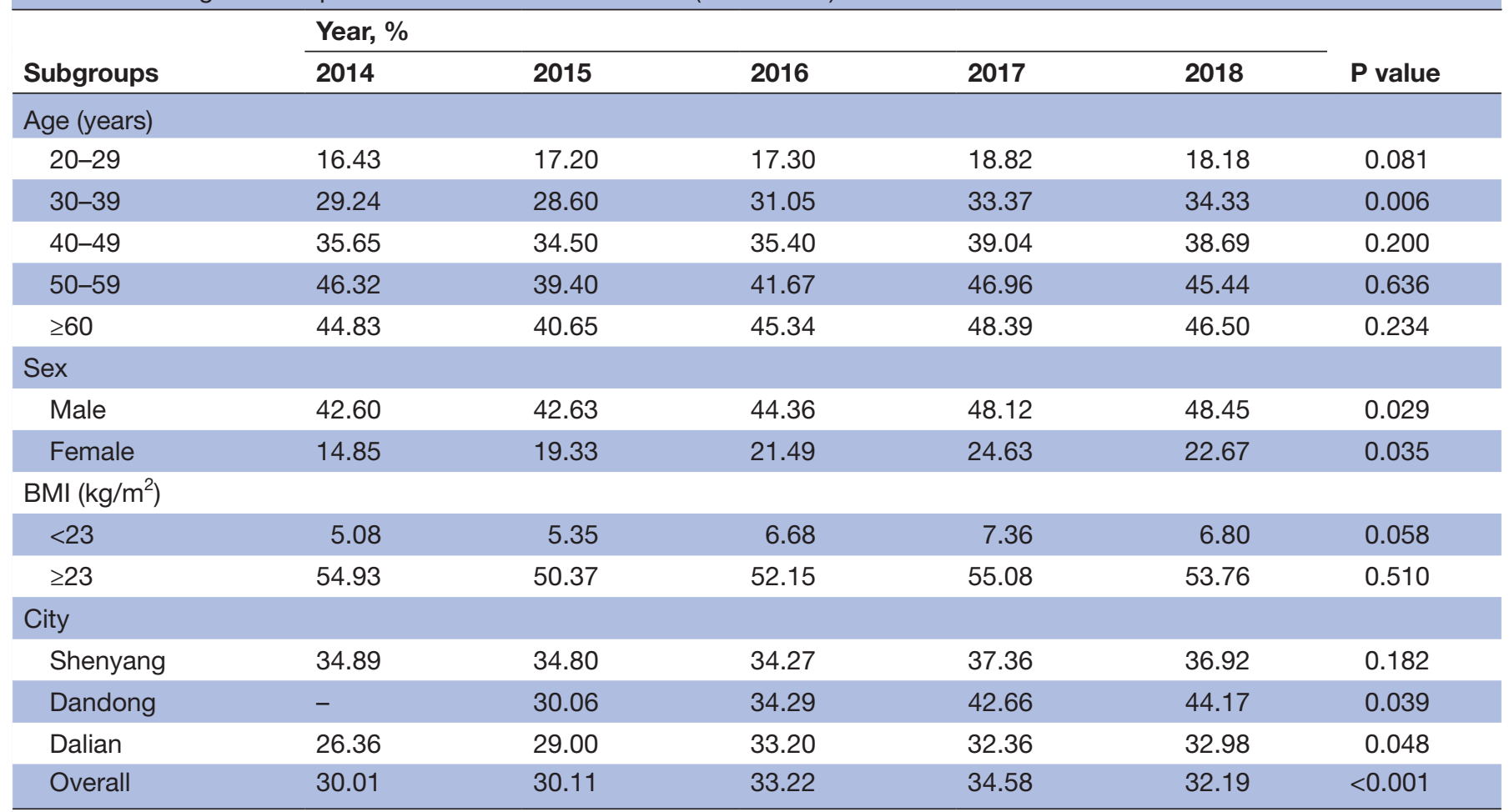

BMI, body mass index; MAFLD, metabolic-associated fatty liver disease.

\section{Prevalence of MAFLD over time}

The total prevalence of MAFLD from 2014 to 2018 was $30.01 \%, 30.11 \%, 33.22 \%, 34.58 \%$ and $32.19 \%$, respectively. The prevalence of MAFLD over the 5 years mainly increased in the age-based group of 30-39 years old $(\mathrm{p}=0.006)$, in men $(\mathrm{p}=0.029)$ and in women $(\mathrm{p}=0.035)$ (table 2). The prevalence of MAFLD in Shenyang over the past 5 years was basically consistent with the general trend in Liaoning. The prevalence of MAFLD in Dandong significantly increased annually over the past 4 years. The prevalence of MAFLD in Dalian substantially increased in 2016, while it declined in 2017 and 2018 (figure 2). The prevalence rate in men and women over the past 5 years

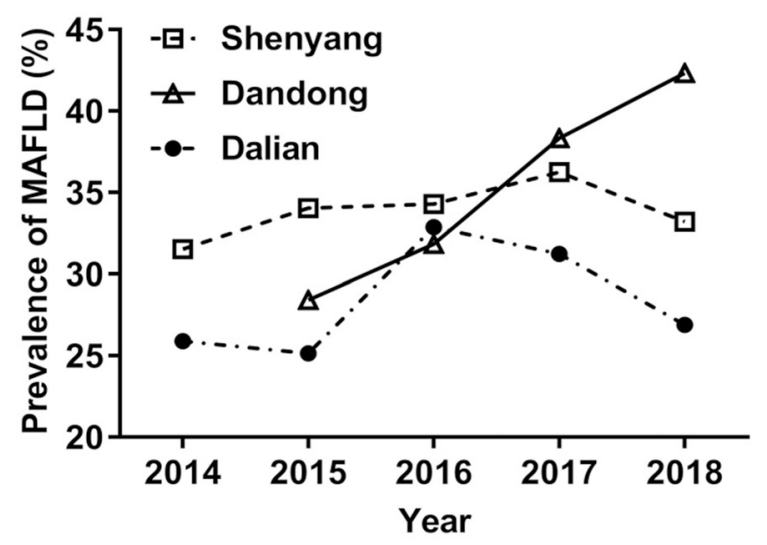

Figure 2 Prevalence of metabolic-associated fatty liver disease (MAFLD) in different cities from 2014 to 2018. Data for Dandong City in 2014 are missing was basically consistent with the general trend of MAFLD in Liaoning (figure 3). The prevalence of MAFLD increases with increase in age in Liaoning.

\section{Biomarkers of MAFLD}

Table 3 presents the biomarkers in all subjects and according to lean/overweight-obese. The number of subjects with overweight/obese MAFLD was 66 609, of whom $3550(5.33 \%)$ were patients with T2DM. The number of cases with lean MAFLD was 5147, of whom 143 $(2.78 \%)$ were patients with T2DM. The number of cases who were diagnosed (for the first time) with FBG level over 7 was 289. Compared with the non-MAFLD group, subjects in the MAFLD group had higher SBP, DBP, FBG,

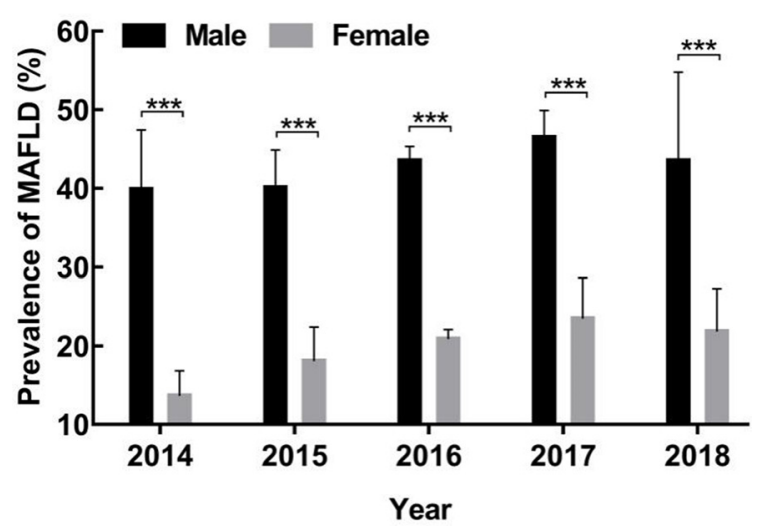

Figure 3 Prevalence of metabolic-associated fatty liver disease (MAFLD) in men is significantly higher than in women (2014-2018). ${ }^{\star \star \star} \mathrm{P}<0.001$. 


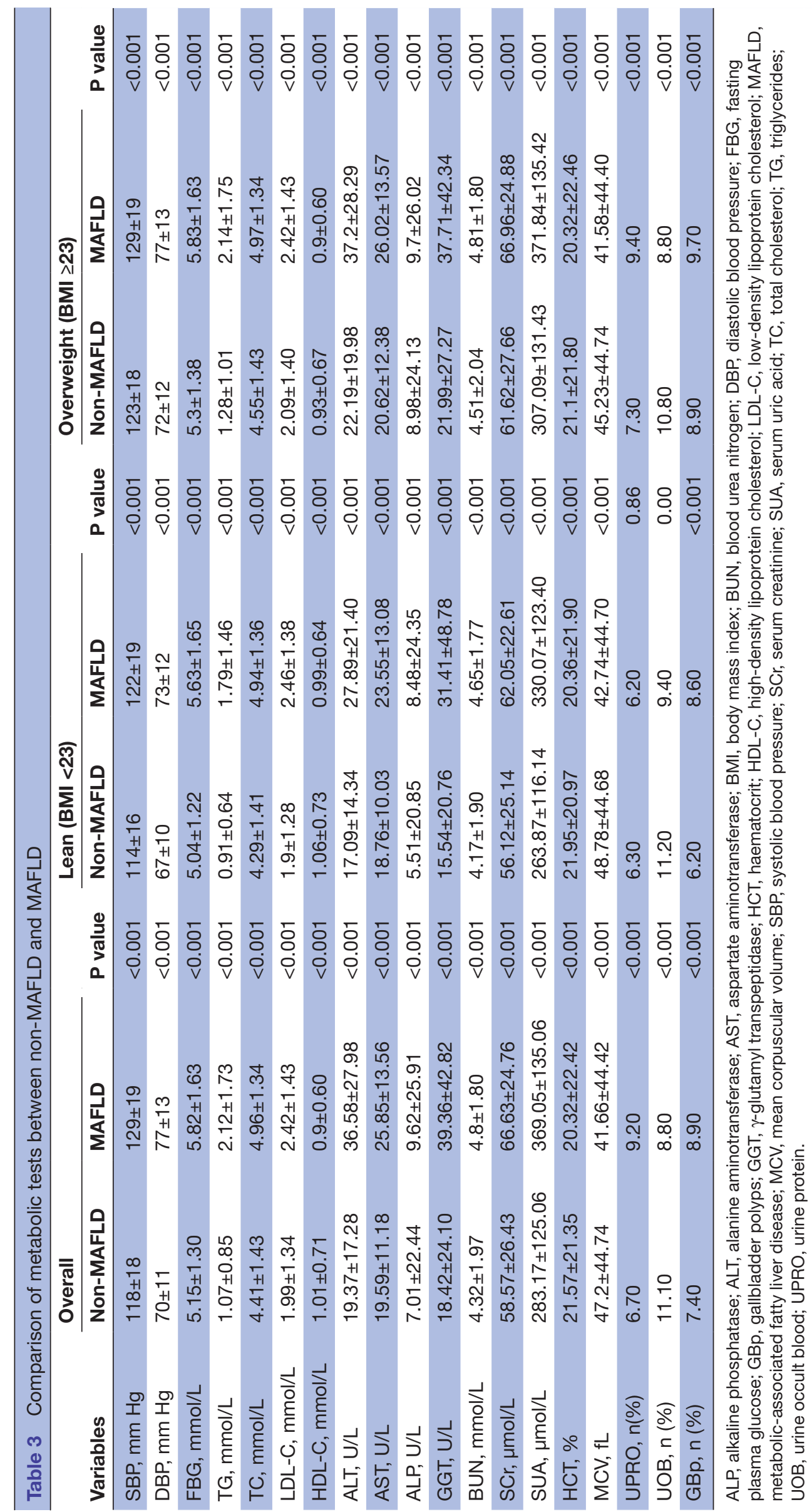


TG, total cholesterol (TC), LDL-C, alanine transaminase (ALT), aspartate transaminase (AST), alkaline phosphatase (ALP), $\gamma$-glutamyl transferase (GGT), blood urea nitrogen (BUN), serum creatinine (SCr) and SUA, higher frequencies of urine protein (UPRO) and gallbladder polyps (GBp), lower HDL-C, haematocrit (HCT) and mean corpuscular volume (MCV), and lower frequency of urine occult blood (UOB) (all $\mathrm{p}<0.001)$. The same tendencies were observed in lean subjects, while there was no significant difference in UPRO $(\mathrm{p}=0.86)$.

\section{MAFLD-associated factors}

Table 4 presents the results of the univariate and multivariate logistic regression analyses of the factors associated with MAFLD. In all subjects, BMI, SBP, DBP, FBG, TG, TC, LDL-C, HDL-C, ALT, AST, ALP, GGT, BUN, SCr, SUA, HCT, MCV and UPRO were independently associated with MAFLD (all $\mathrm{p}<0.001)$, while UOB $(\mathrm{p}=0.47)$ and GBp $(\mathrm{p}=0.21)$ were not. In lean subjects, DBP, FBG, TG, TC, LDL-C, HDL-C, AST, ALP, GGT, SCr, SUA, HCT and MCV were independently associated with MAFLD (all $\mathrm{p}<0.001)$, while SBP $(p=0.51)$, ALT $(p=0.27)$, BUN $(p=0.16)$, UPRO $(\mathrm{p}=0.57)$, UOB $(\mathrm{p}=0.06)$ and $\mathrm{GBp}(\mathrm{p}=0.06)$ were not.

\section{DISCUSSION}

The present study showed that the prevalence of MAFLD in Shenyang, Dandong and Dalian cities varied, and higher BMI value and age played significant roles in the development of the disease. In addition, biomarkers such as DBP, FBG, TG, LDL-C, ALT, GGT, BUN, SUA, HCT, UPRO, and GBp were independently and positively correlated with the prevalence of MAFLD, whereas HDL-C and MCV were negatively correlated. Therefore, the prevalence of MAFLD in Liaoning was related to sex, cities with different economic statuses, BMI and multiple metabolic indicators.

The increasing trend in the prevalence of NAFLD follows the level of industrialisation and urbanisation. At present, China is the fastest-growing major economy in the world, and there are problems associated with a Westernised diet, sedentary lifestyle and metabolism, which are correlated with MAFLD. Thus, there is a closer relationship between MAFLD and economy than NAFLD. Studies have shown that the increase in the total annual NAFLD prevalence in China is consistent with the improvement in the gross domestic product (GDP) per capita. ${ }^{20}$ An increase in morbidity in various regions of mainland China could be related to the increase in GDP per capita, while areas with the highest GDP per capita $(\geq \$ 13000)$ did not exhibit an increased incidence of NAFLD. ${ }^{21}$ According to the National Bureau of Statistics of China, the GDP per capita of Liaoning Province has ranked first in northeast China over the past 5 years, whereas it is still in the lower-middle level nationally. The present study revealed that the prevalence of MAFLD in Liaoning (35.1\%) was slightly higher than the overall prevalence in China $(29.2 \%) .{ }^{12}$ The current study selected two economically developed cities (Dalian and Shenyang) and one city with a moderate development (Dandong). The prevalence of MAFLD in these three cities was inversely proportional to the level of urban economic development. The prevalence of MAFLD in Dalian was lower than that in Shenyang. This could be related to the fact that Dalian is a coastal city, with dietary habits different from those of inland cities.

Differences in age were also confirmed. The prevalence of MAFLD in the age-based group of 20-29 years old was $17.9 \%$, while it was $45.6 \%$ in the age-based group of $>60$ years old, which is in agreement with previous studies which demonstrated that age could play a crucial factor in the prevalence of NAFLD. ${ }^{11} 1215$

The present study indicated that the overall prevalence of MAFLD in men was higher than in women, which is basically consistent with the previously reported findings in other regions of China. ${ }^{22}$ The prevalence of MAFLD in middle-aged men was the highest and reached the peak at $40-49$ years old $(50.27 \%)$. This high prevalence might be related to high stress, irregular work and rest, and decreased metabolism among middle-aged men. The prevalence of MAFLD in women aged over 50 years old was significantly higher, which could be related to the age range for menopause.

Obesity is closely associated with metabolic diseases, such as MAFLD. The current study confirmed that BMI $\geq 23 \mathrm{~kg} / \mathrm{m}^{2}$ is an independent risk factor for MAFLD in Liaoning. Therefore, for overweight and obese individuals, it is necessary to improve diet and exercise management, even using drugs or surgery, if required. It has been reported that the global NAFLD prevalence in lean population was $5 \%-26 \% .{ }^{23}$ In the present study, the prevalence of NAFLD in lean population was $10.75 \%$, which is similar to previously reported results. ${ }^{24}$

In this study, both lean MAFLD and non-lean MAFLD were closely related to metabolic indicators. Those metabolic indicators were also associated with T2DM, metabolic syndrome, obesity and liver diseases, as supported by previous studies. ${ }^{11} 1215$ 22-24 In addition to obesity, variations in the prevalence of MAFLD follow the epidemic trends of T2DM and metabolic syndrome (MetS). The prevalence of MAFLD in patients with hyperlipidaemia and T2DM is higher, reaching $27 \%-92 \%$ and $28 \%-70 \%$, respectively. Patients with MAFLD mainly have hyperlipidaemia, hypertension, T2DM and metabolic syndrome. ${ }^{25}$ In the current study, multivariate logistic regression analysis found that DBP, TG, LDL-C, FBG and SUA were independent risk factors for MAFLD, while HDL-C was independently associated with MAFLD. Therefore, additional attention should be paid to hyperlipidaemia, hypertension and T2DM to assess their influence on the prevention and treatment of MAFLD. Regarding the correlation between SUA and MAFLD, SUA elevation was reported as one of the risk factors for MAFLD. ${ }^{26}$

Although genetic susceptibility was not examined in the current study, it is involved in NAFLD. Polymorphisms in PNPLA3, ${ }^{27}$ SREBF-2, ${ }^{28}$ CETP $^{28}{ }^{29}$ and APOC3 ${ }^{30}$ have 
Table 4 Multivariable analyses of the factors associated with MAFLD

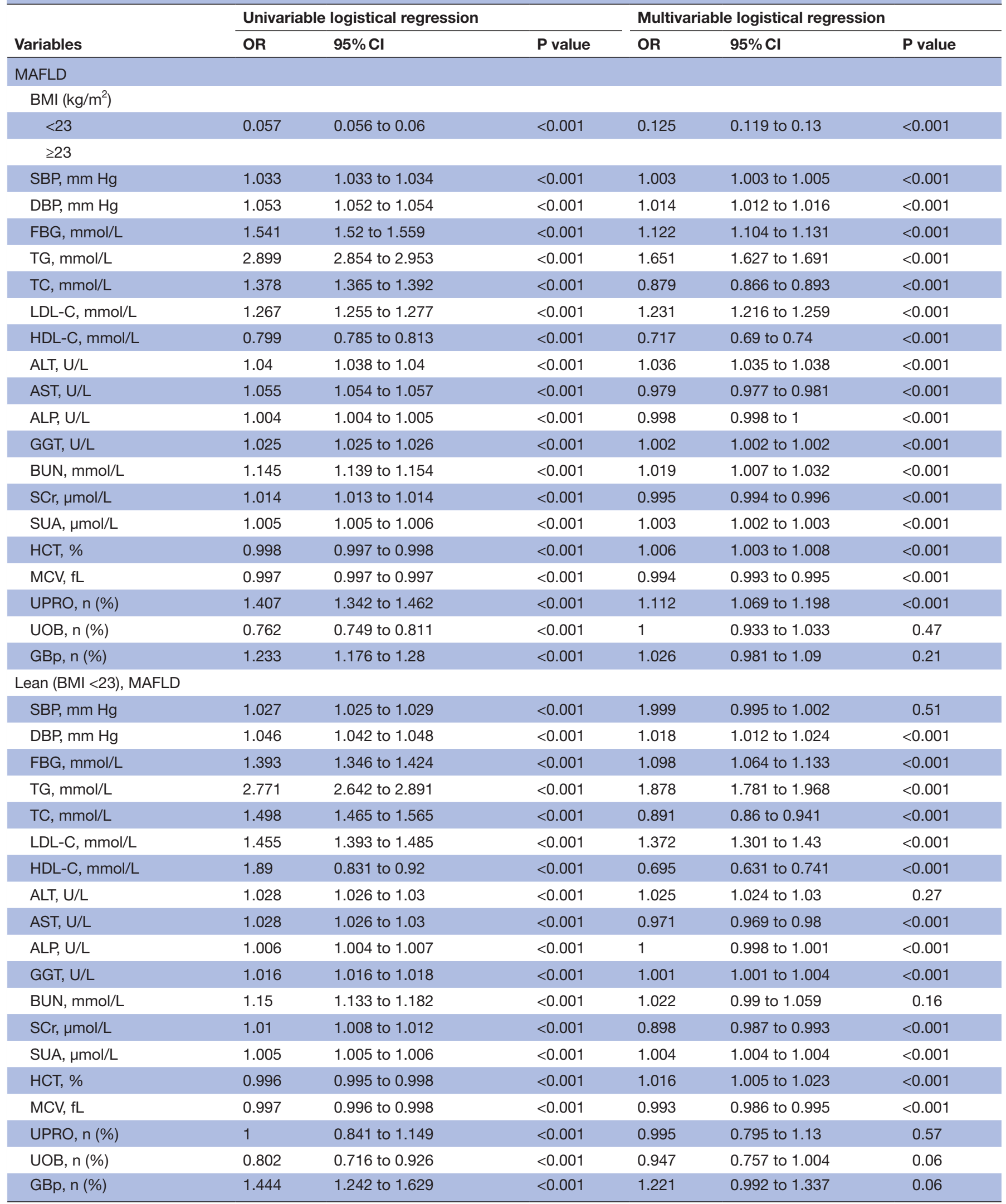

ALP, alkaline phosphatase; ALT, alanine aminotransferase; AST, aspartate aminotransferase; BMI, body mass index; BUN, blood urea nitrogen; DBP, diastolic blood pressure; FBG, fasting plasma glucose; GBp, gallbladder polyps; GGT, $\gamma$-glutamyl transpeptidase; HCT, haematocrit; HDL-C, highdensity lipoprotein cholesterol; LDL-C, low-density lipoprotein cholesterol; MAFLD, metabolic-associated fatty liver disease; MCV, mean corpuscular volume; SBP, systolic blood pressure; SCr, serum creatinine; SUA, serum uric acid; TC, total cholesterol; TG, triglyceride; UOB, urine occult blood; UPRO, urine protein. 
been found to be associated with lean NAFLD. In addition to genetic polymorphisms, lean NAFLD cases have increased bile acid and Farnesoid X-Activated Receptor (FXR) activity due to metabolic abnormalities and changes in intestinal microbial composition. ${ }^{31} 32$ These factors should be assessed in future studies.

Regarding diabetes, because 3156 patients had previously received systemic treatment for diabetes, the current blood glucose levels and metabolic indicators were in the normal range; thus, in order to reduce error in the analysis of risk factors, we excluded these patients. In addition, patients with diabetes analysed in the present study were previously diagnosed, of whom 289 patients were found to have elevated FBG levels for the first time. As physical examinations did not involve Oral Glucose Tolerance Test (OGTT) and repeated tests, we did not classify such patients as diabetic.

This study has some limitations. Although the examinations were performed at the same clinic, they were conducted at three different physical locations over the past 5 years. Bias due to the different locations and changes in practice over time could not be excluded. Due to the large number of physical examinations and no measurement of waist circumference and high-sensitivity $\mathrm{C}$ reactive protein, the diagnosis of MAFLD in some patients with normal BMI might be ignored. In addition, ultrasound is operator-dependent and bias in the diagnosis of MAFLD could not be excluded. Finally, this was a cross-sectional study that could not provide any causal relationship between MAFLD and the associated factors.

In conclusion, the prevalence of MAFLD in Liaoning was found to be associated with sex, cities with different economic statuses, BMI and multiple metabolic indicators. Longitudinal studies are necessary to further determine factors associated with the development of MAFLD.

Contributors Conception and design: LG, XZ, YL. Administrative support: JS, DL, YL. Provision of study materials or patients: LG, XJ, NW. Collection and assembly of data: HT, XW, ZZ. Data analysis and interpretation: HF, JL. Manuscript writing: all authors. Final approval of the manuscript: all authors. Guarantor: YL.

Funding The authors have not declared a specific grant for this research from any funding agency in the public, commercial or not-for-profit sectors.

Competing interests None declared.

Patient and public involvement Patients and/or the public were not involved in the design, or conduct, or reporting, or dissemination plans of this research.

Patient consent for publication Not required.

Ethics approval This study involves human participants and was conducted in accordance with the Declaration of Helsinki. The protocol was approved by the Ethics Committee of the First Hospital of China Medical University ((2020)2020257-2). The ethics committee waived the requirement for informed consent due to the retrospective nature of the study. Participants gave informed consent to participate in the study before taking part.

Provenance and peer review Not commissioned; externally peer reviewed.

Data availability statement Data are available upon reasonable request.

Open access This is an open access article distributed in accordance with the Creative Commons Attribution Non Commercial (CC BY-NC 4.0) license, which permits others to distribute, remix, adapt, build upon this work non-commercially, and license their derivative works on different terms, provided the original work is properly cited, appropriate credit is given, any changes made indicated, and the use is non-commercial. See: http://creativecommons.org/licenses/by-nc/4.0/.
ORCID iD

Yiling Li http://orcid.org/0000-0003-3209-8105

\section{REFERENCES}

1 Younossi ZM, Koenig AB, Abdelatif D, et al. Global epidemiology of nonalcoholic fatty liver disease-Meta-analytic assessment of prevalence, incidence, and outcomes. Hepatology 2016;64:73-84.

2 Stål P. Liver fibrosis in non-alcoholic fatty liver disease - diagnostic challenge with prognostic significance. World J Gastroenterol 2015;21:11077-87.

3 Eslam M, Sanyal AJ, George J, et al. MAFLD: a Consensus-Driven proposed nomenclature for metabolic associated fatty liver disease. Gastroenterology 2020;158:1999-2014.

4 Fouad Y, Waked I, Bollipo S, et al. What's in a name? Renaming 'NAFLD' to 'MAFLD'. Liver Int 2020;40:1254-61.

5 Pai RK. Nafld histology: a critical review and comparison of scoring systems. Curr Hepatol Rep 2019;18:473-81.

6 Vernon G, Baranova A, Younossi ZM. Systematic review: the epidemiology and natural history of non-alcoholic fatty liver disease and non-alcoholic steatohepatitis in adults. Aliment Pharmacol Ther 2011;34:274-85.

7 Chalasani N, Younossi Z, Lavine JE, et al. The diagnosis and management of non-alcoholic fatty liver disease: practice guideline by the American gastroenterological association, American association for the study of liver diseases, and American College of gastroenterology. Gastroenterology 2012;142:1592-609.

8 Non-Alcoholic Fatty Liver Disease. Non-Alcoholic fatty liver disease: assessment and management. London: National Institute for Health and Care Excellence, 2016.

9 Rinella ME. Nonalcoholic fatty liver disease: a systematic review. JAMA 2015;313:2263-73.

10 Shen FF, Lu LG. Advances in noninvasive methods for diagnosing nonalcoholic fatty liver disease. J Dig Dis 2016;17:565-71.

11 Zhou J, Zhou F, Wang W, et al. Epidemiological features of NAFLD from 1999 to 2018 in China. Hepatology 2020;71:1851-64.

12 Zhou F, Zhou J, Wang W, et al. Unexpected rapid increase in the burden of NAFLD in China from 2008 to 2018: a systematic review and meta-analysis. Hepatology 2019;70:1119-33.

13 Caballería L, Auladell MA, Torán P, et al. Risk factors associated with non-alcoholic fatty liver disease in subjects from primary care units. A case-control study. BMC Gastroenterol 2008;8:44.

14 Hartmann P, Schnabl B. Risk factors for progression of and treatment options for NAFLD in children. Clin Liver Dis 2018;11:11-15.

15 Wu Y, Zheng Q, Zou B, et al. The epidemiology of NAFLD in mainland China with analysis by adjusted gross regional domestic product: a meta-analysis. Hepatol Int 2020;14:259-69.

16 Dulai PS, Singh S, Patel J, et al. Increased risk of mortality by fibrosis stage in nonalcoholic fatty liver disease: systematic review and metaanalysis. Hepatology 2017;65:1557-65.

17 WHO Expert Consultation. Appropriate body-mass index for Asian populations and its implications for policy and intervention strategies. Lancet 2004;363:157-63.

18 Eslam M, Newsome PN, Sarin SK, et al. A new definition for metabolic dysfunction-associated fatty liver disease: an international expert consensus statement. J Hepatol 2020;73:202-9.

19 Mahale AR, Prabhu SD, Nachiappan M, et al. Clinical relevance of reporting fatty liver on ultrasound in asymptomatic patients during routine health checkups. J Int Med Res 2018;46:4447-54.

20 Cai J, Zhang X-J, Li H. Progress and challenges in the prevention and control of nonalcoholic fatty liver disease. Med Res Rev 2019;39:328-48.

21 Fan J-G, Kim S-U, Wong VW-S. New trends on obesity and NAFLD in Asia. J Hepatol 2017;67:862-73.

22 Zhu J-Z, Zhou Q-Y, Wang Y-M, et al. Prevalence of fatty liver disease and the economy in China: a systematic review. World $\mathrm{J}$ Gastroenterol 2015;21:5695-706.

23 Wang AY, Dhaliwal J, Mouzaki M. Lean non-alcoholic fatty liver disease. Clin Nutr 2019;38:975-81.

24 Shi $Y$, Wang $Q$, Sun Y, et al. The prevalence of Lean/Nonobese nonalcoholic fatty liver disease: a systematic review and metaanalysis. J Clin Gastroenterol 2020;54:378-87.

25 Fan JG, Wei L, Zhuang H, et al. Guidelines of prevention and treatment of nonalcoholic fatty liver disease (2018, China). J Dig Dis 2019;20:163-73.

26 Ma Z, Xu C, Kang X, et al. Changing trajectories of serum uric acid and risk of non-alcoholic fatty liver disease: a prospective cohort study. J Trans/ Med 2020;18:133.

27 Musso G, Cassader M, Bo S, et al. Sterol regulatory element-binding factor 2 (SREBF-2) predicts 7-year NAFLD incidence and severity of 
liver disease and lipoprotein and glucose dysmetabolism. Diabetes 2013;62:1109-20.

28 Adams LA, Marsh JA, Ayonrinde OT, et al. Cholesteryl ester transfer protein gene polymorphisms increase the risk of fatty liver in females independent of adiposity. J Gastroenterol Hepatol 2012;27:1520-7.

29 Petersen KF, Dufour S, Hariri A, et al. Apolipoprotein C3 gene variants in nonalcoholic fatty liver disease. $N$ Engl $\mathrm{J}$ Med 2010;362:1082-9.

30 Li C, Guo P, Okekunle AP, et al. Lean non-alcoholic fatty liver disease patients had comparable total caloric, carbohydrate, protein, fat, iron, sleep duration and overtime work as obese non-alcoholic fatty liver disease patients. $J$ Gastroenterol Hepatol 2019;34:256-62.

31 Safari Z, Gérard P. The links between the gut microbiome and non-alcoholic fatty liver disease (NAFLD). Cell Mol Life Sci 2019;76:1541-58.

32 Suk KT, Kim DJ. Gut microbiota: novel therapeutic target for nonalcoholic fatty liver disease. Expert Rev Gastroenterol Hepatol 2019;13:193-204. 